\title{
INTIMIDADES CONECTADAS. REFLEXIONES SOBRE UN CUARTO PROPIO CONECTADO, DE REMEDIOS ZAFRA ${ }^{1}$
}

\author{
CONNECTED PRIVACIES. ANALYSIS OF \\ REMEDIOS ZAFRA'S UN CUARTO PROPIO CONECTADO
}

Nadia MÉKOUAR-HERTZBERG

Universidad de Pau et des Pays de l'Adour

nadia.mekouar@univ-pau.fr

Resumen: Proponemos una lectura de Un cuarto propio conectado (Zafra), centrado en la cuestión de la intimidad en el contexto de desarrollo acelerado e irreversible de Internet. En la estela de Woolf, el cuarto propio sigue siendo un lugar propicio para la emergencia de intimidades femeninas y feministas, centros de gestión del yo y de las relaciones de poder. Sin embargo, la dinámica y la forma de esa intimidad evolucionan bajo el impulso de la digitalización. Veremos cómo, a medio camino entre ensayo, autobiografía y cuento, el texto de Zafra configura una nueva cartografía de la intimidad, de sus fronteras y de sus funciones.

Palabras clave: Un cuarto propio conectado. Remedios Zafra. Intimidad. Digitalización. Feminismo.

Abstract: We propose an analysis of Un cuarto propio conectado (Zafra), focused on the issue of privacy in the context of the accelerated and irreversible development of the Internet. In the wake of Woolf, the notion of a room of one's own continues to be the very place where the

\footnotetext{
${ }^{1}$ Este artículo se inscribe en el marco del proyecto de investigación franco-español "Figures et frontières de l'intime à l'époque contemporaine / Figuras y fronteras de la intimidad en la época contemporánea" (OPE-2017-0042), de la Universidad de Pau et des Pays de l'Adour EFM (FR 4153), ALTER (EA7504), ITEM (EA 3002) en colaboración con el grupo de investigación "Lenguajes" (HUM 224) de la Universidad de Córdoba (España) y el laboratorio "Arts Plastiques" (EA 7472) de la Universidad de Rennes 2 (Francia).
} 
emergence of feminine and feminist intimacies becomes possible, a center of management for the self and power relationships. However, the dynamics and form of intimacy have evolved through digitalization. We will see how, halfway between an essay, an autobiography and a short story, Zafra's text maps out a new cartography of intimacy, its borders and its functions.

Key Words: Un cuarto propio conectado. Remedios Zafra. Intimacy. Digitalization. Feminism.

En 2010, R. Zafra publica Un cuarto propio conectado. (Ciber) espacio y (auto)gestión del yo (Zafra, 2010), verdadero ovni literario cuyo contenido se inscribe, sin embargo, en un conjunto de nociones familiares como identidad, subjetividad, sujeto, persona e intimidad. El texto tiene también en cuenta el mayor trastorno que conocen las sociedades en la actualidad: la revolución digital que impacta en todos los aspectos de nuestra vida cotidiana, de nuestro espacio político, económico, lingüístico, cultural y artístico. En ese contexto, la recuperación del título de Virginia Woolf no tiene solo como proyecto proponer una exégesis actualizada y adaptada a nuestra contemporaneidad del famoso texto: se trata de una forma de llevarnos a preguntarnos ¿qué nos diría esa obra si naciera ahora?, ¿cuál es el impacto de la digitalización creciente de las existencias en la forma de concebir y de formular nuestras intimidades?, ¿cómo compaginar el principio de la conexión, cada vez más omnipresente, con esa categoría tradicionalmente ubicada en el mundo subjetivo, fruto de cierto apartamiento o, cuando no, de perseverante encierro?

Lo íntimo, literalmente, lo más interior del adentro, designa esta zona no solo privada sino también reservada, la parte supuestamente más profunda y secreta, donde el sujeto mora y se experimenta a sí mismo, fuera de la mirada del otro. A partir de esa realidad semántica y etimológica, la noción ha conocido muchas evoluciones, confirmando su calidad de construcción social e histórica, subordinada a las numerosas concepciones de la categoría de sujeto que han ido surgiendo a lo largo de los siglos, en las sociedades occidentales en particular. No obstante, el Cuarto propio conectado, no es una variante más. Subraya ante todo una profunda e 
irreversible ruptura, un "obstáculo epistemológico"2 que no se refiere a la intimidad propiamente dicha, a lo "propio" sino, en primer lugar, a espacios, sitios y demás cuartos que son los escenarios privilegiados de la emergencia de esa intimidad. ¿Cuáles son las nuevas topografías de la intimidad en unos espacios cuyas fronteras no dejan de desplazarse, sin desaparecer nunca, en unos espacios conectados e interconectados, transitados por sujetos solitarios en su cuarto?

Tal redistribución de los espacios, en el seno de prácticas sociales profundamente transformadas por el uso de las herramientas digitales, es un componente central de Un cuarto propio conectado. Esa obra inclasificable adopta un sistema discursivo que renuncia a la homogeneidad y a la linealidad, mimético, en cierta medida, de ese espacio íntimo fronterizo, dinamizado —idinamitado? - por intercambios e intersecciones constantes y, sin embargo, claramente cartografiable. Esa nueva cartografía será un aspecto fundamental de las reflexiones que siguen y nos permitirá observar las nuevas configuraciones del cuarto propio y sus impactos en la fabricación de una intimidad abierta, implicada y feminista que rehabilita abiertamente la preeminencia de un sujeto céntrico.

\section{LA INTIMIDAD DE LA NETIANA}

¿Qué es lo que estamos leyendo? Es seguramente una de las primeras preguntas que nos hacemos al abrir el volumen. El índice del texto, constituido de títulos inesperados y enigmáticos ("Capítulo piel"; "Capítulo de luz baja", etc.) deja de cumplir con su tradicional función de guía orientadora por el texto. Sin embargo, una serie de términos florecen, en forma de nube de palabras: cuarto, conectado, virtual, yo, yoes, visibilidad, pre-capitalismo visual, online, jugar, database, listas, imagen, real, ventana, avatares, espacio, ver, no ver, ciberespacio, cuerpos, multiplicidad que nos invita intuitivamente a asociar intimidad con espacio, introspección con relación, identificación con invención, identidad con pluralidad, en el marco de un contexto digitalizado. Igualmente, la organización gráfica del texto, entrecortada de fragmentos con caracteres repentinamente más grandes o más pequeños, nos proyecta en una dimensión digital alimentada por los nuevos soportes tecnológicos,

2 Recordamos que la expresión fue propuesta por Gaston Bachelard en Le nouvel esprit scientifique. 
a la vez que nos confirma la indisoluble vinculación entre conexión digital e intimidad. La apertura del libro con una cita del micro-poema de Laura Bey, Mi vida en la primera IP (concebido con la propia R. Zafra, y aún sin publicar), es ya programática de una elaboración de la intimidad irremediablemente conectada e ilustrativa de la emergencia de un texto digitalizado:

La Red ha dividido mi vida en 300 tareas, de las cuales 98 tienen que ver con teclear; 35 corresponden a ;buscar, buscar!; 6 a actualizar software; 51 a almacenar archivos; 67 a minimizar-maximizar; 18 a descubrir mi cuerpo (ioh, cielos, mi cuerpo!); 34 a esperar que llegue "ese" correo; 19 a derivar online; 45 a contactar contigo; contigo; contigo; 36: "do it yourself" (myself); 21 a "no me están viendo"; 9 a "que mañana será otro día". La Red no rehúsa otras tareas heterogéneas para hacer en la intimidad de mi cuarto propio. La suma no coincide con la división prevista porque constantemente surgen tareas y clasificaciones nuevas (Zafra, 2010: 10).

Las copias de pantallas, como el delicioso "Verify you are not a robot" (Zafra, 2010: 18) o la inserción directa de fórmulas como "(x_/l)" (Zafra, 2010: 19), elementos de sintaxis de lenguaje informático, nos confirman, definitivamente, que Un cuarto propio conectado, en tanto que objeto para ser manipulado y leído, organiza una inédita y astuta confluencia entre libro y pantalla. La pantalla no sustituye al libro, sino que irrumpe en él; el libro acoge, "captura" una pantalla repentinamente dotada del estatuto de texto literario en sí.

Sin lugar a dudas, esos procedimientos participan del rechazo a la "linealidad y la disciplinariedad" (Zafra, 2010: 13) presente y afirmado en Un cuarto propio conectado pero, también, característico de otros textos de R. Zafra. De la misma manera, las vacilaciones genéricas entre ensayo y autobiografía, novela y cuento, con claras afinidades con el manifiesto político o el texto de ciencia ficción, sitúan el texto en un no man's land literario, que responde a una verdadera preocupación por parte de la autora, y a un auténtico desafío: la necesidad de ajustar la reflexión a la emergencia de un dispositivo revolucionario, la irrupción de Internet y, con él, de un nuevo modo de relacionarse. Esta necesidad constituía ya una línea de fuerza del ensayo Netianas. $N(h)$ acer mujer en Internet y fue 
expresada por la propia autora en varias ocasiones:

Creo que toda reflexión crítica contemporánea debe habitar la dificultad de su tiempo, e Internet forma parte de esa dificultad, en ella se generan nuevas preguntas sobre lo que somos y lo que podemos llegar a ser en una sociedad cada vez más mediada por pantallas. [...]. La falta de reflexión crítica supone una aceptación de lo que viene dado y, posiblemente, una reproducción de las formas de poder y relación que ya hemos visto en el pasado. Sería por tanto una oportunidad para nuestra imaginación plantearnos qué somos en Internet y qué queremos ser (Zafra, 2005b).

La sociedad en red impacta en nuestra forma de trabajar pero, también, en nuestra forma de relacionarnos con otras personas, con nosotros mismos, con "lo que somos en Internet y lo que queremos ser". En otros términos, es imposible pensar nuestro ser en su singularidad sin Internet $\mathrm{y}$, por ende, sin la dinámica relacional constitutiva de Internet. La toma de partido adisciplinar y agenérica por parte de la autora responde, por consiguiente, a la emergencia de ese espacio nuevo en que se encuentra y se define el sujeto; la innovación formal es el corolario necesario de la conceptualización de una nueva figura, de un sujeto online que, en y por el proceso mismo de su devenir digital (de su conexión), tiene que plantearse la pregunta de qué es y de qué quiere ser, para habitar plenamente el nuevo espacio virtual al que tiene acceso. En este dispositivo, la netiana es este sujeto online y en femenino, figura en que confluyen las problemáticas relacionadas con el espacio virtual por una parte y con las praxis femeninas y feministas por otra: las mujeres tienen que habitar el espacio virtual, apoderarse de la tecnología que le es consustancial, para inventar y negociar nuevas relaciones de género. En Un cuarto propio conectado, esa apropiación toma la forma de una verdadera requisición del espacio virtual como territorio de la intimidad. El texto puede ser leído como la consagración de la figura de la netiana para quien la pantalla se presenta como "hogar íntimo" (Zafra, 2005a: 39). Para ella, el espacio virtual se convierte en el espacio por excelencia de la intimidad, su atributo moderno, dotado de una sacralidad renovada e inesperada:

Un espacio a veces contemplativo y místico, y siempre solitario 
para el cuerpo. [...] un jardín secreto donde podemos permitirnos ser: más repetitivos, más diferentes, más auténticos, más libres que lo que la vida - off line-del cuerpo y los convencionalismos nos permite. En este jardín secreto que proyectamos en la pantalla, donde pareciera que reubicamos los antiguos espacios de rezo y búsqueda interior, toman forma unas nuevas "santas", inmateriales, latentes netianas (Zafra, 2005a: 39).

Por cierto, R. Zafra no cae en la trampa de una visión idílica y angélica de Internet: "el lugar alternativo (jardín secreto) de Internet no está exento de riesgos, de hecho ese "ser otro" no tiene por qué suponer una liberación de los estereotipos y prejuicios sobre el cuerpo del mundo físico [...]" (Zafra, 2005a: 41). Este "jardín secreto" digital es el receptáculo de convencionalismos y de normas (en particular de las normas de género) que consigue reproducir y transmitir con una capacidad de amplificación asombrosa e inquietante. No obstante, en este dispositivo, las netianas son las que, potencialmente, van adquiriendo la capacidad de desprenderse de esos estereotipos, las que se mantienen en esta línea de cresta incómoda $\mathrm{y}$, sin embargo, fructífera, de interiorización de los cánones para mejor subvertirlos. De esta forma, la pantalla puede convertirse, en un mismo movimiento, en espacio de subversión, de deconstrucción por una parte $\mathrm{y}$, por otra, de elaboración de un fuero interior auténtico, de esa zona superlativamente interior de nuestros adentros. En este sentido, podríamos decir que Internet abre la posibilidad de un espacio de intimidad altamente irónico, siendo concebida la ironía como esa modalidad de "distanciamiento constructivo" claramente formulada por el filósofo V. Jankelevitch: "L'ironie c'est l'inquiétude de la vie inconfortable [...]. L'ironie proteste contre la rationalisme statique et rend hommage à la temporalité de la vie; l'ironie dit à sa manière que toute essence de l'être est le devenir, qu'il n'y a pas d'autre manière d'être que de devoir-être" (Jankelevitch, 1979: 183) ${ }^{3}$. La intimidad conectada descansa, o puede descansar, en este postulado de "distanciamiento constructivo" mediante el cual la netiana consigue abrir varios "puntos de fuga del sujeto que, sin alienarlo, lo

${ }^{3}$ La ironía es la inquietud de la vida incómoda [...]. La ironía protesta contra el racionalismo estático y rinde homenaje a la temporalidad de la vida; la ironía dice a su manera que la esencia del ser es el devenir, que no hay otra forma de ser sino el "tener que ser". (La traducción es nuestra). 
modifican, lo exceden o lo anulan" (Zafra, 2005a: 43): intimidad "irónica" que se impone como una construcción distanciada y plural, en el marco de un espacio conectado que R. Zafra califica de "fluido" (Zafra, 2010: 53). Un espacio cuyas fronteras son líneas abiertas, inciertas y movedizas, promesas de una dinámica de constante renovación, de una "inquietud" saludable y necesaria.

Esta netiana es la protagonista implícita de un Cuarto propio conectado. Se apropia de un espacio doblemente propicio para una expresión de la intimidad que "excede al sujeto", que le modifica "sin alienarlo": un cuarto "en red" que posibilita la creación de una intimidad online y cuya particularidad notable es que deja de ser una dimensión fuera de la mirada del otro. Al contrario, es un proceso que se efectúa debajo de esta mirada. La intimidad online es una intimidad especular: se fabrica en y por la mirada del/de la otro/otra.

\section{LA INTIMIDAD COMPROMETIDA}

Si Un cuarto propio conectado contesta a las nuevas preguntas planteadas por la emergencia de Internet, es también la plasmación de una serie de pensamientos sobre la noción de intimidad y puede ser leído como un verdadero crisol, un compendio de un conjunto nocional previo que recupera con mucha habilidad.

Se apoya primero en una tradición conceptual que relaciona las nociones de intimidad, feminismo y creación a través de una topología doméstica centrada en el motivo del cuarto propio ${ }^{4}$. En la perspectiva de esa filiación, el cuarto propio de V. Woolf es el punto de partida explícito del texto de R. Zafra. En ambos textos, comprobamos la misma vacilación entre ensayo y confesión, reflexión y recuerdo pero, sobre todo, la misma correlación entre intimidad/feminismo/subjetividad creadora: o sea, entre 1/ la emergencia de una psique propia, singularizada por sus afectos, pensamientos o sentimientos más interiores; 2/ la actuación política de sujetos femeninos a favor de la transformación de las relaciones de género; y 3/ la promoción de esos sujetos como sujetos creadores (y no

${ }^{4}$ En el campo de la literatura contemporánea española, El cuarto de atrás de Carmen Martín Gaite es de particular relevancia para ilustrar esta importancia del espacio doméstico en la configuración y la expresión de la intimidad femenina. 
solo procreadores), capaces de discursos (estéticos, éticos, políticos) autónomos. La perpetuación de ese tríptico en el cuarto propio conectado es claramente apuntada por R. Zafra:

[...] el espacio de intimidad que $V$. Woolf reclamaba como crucial para el desarrollo intelectual de las mujeres sigue siendo un lugar singular para la reivindicación creativa pero también para reflexión política, ampliada hoy a la comprensión de las nuevas dinámicas económicas a través de la pantalla conectada a la red (Zafra, 2010: $52)$.

En otros términos, si bien el cuarto propio conectado ilustra una "ruptura epistemológica" profunda, si bien es un espacio "resemantizado", según los propios término de R. Zafra, sigue siendo, en adecuación con el texto de Woolf, un espacio fundador de la "autogestión" de un yo femenino, feminista y creador: en ambos textos, el espacio doméstico deja de ser un espacio de exclusión y de asignación para convertirse en un espacio fronterizo, susceptible de gestar nuevas formas de poder a través de una redefinición del yo femenino así como de sus potencialidades emancipatorias y creadoras.

De forma más general, Un cuarto propio conectado se inscribe en una concepción de la intimidad y de lo íntimo como nociones que superan la tradicional oposición interior/exterior. El cuarto propio conectado es un espacio "[...] donde aislamiento no deviene autismo, sino formas de espacialización y colectividad que amplían las posibilidades de relación, autonomía y optimización de tiempos y energías, pero también la viabilidad de vidas paralelas" (Zafra, 2010: 31). En esta perspectiva, hay que recordar las dos acepciones posibles y concomitantes que se han desarrollado a partir de la etimología latina, el superlativo latín intimus ("lo más interior"): por una parte, lo íntimo como la zona más profunda y secreta, donde el sujeto mora, fuera de la mirada del otro; por otra parte, lo íntimo como lo que se asocia más profundamente al otro, lo que se comparte con un amigo íntimo. Finalmente, lo íntimo designa un espacio protegido, reservado e inasequible, pero puede también suponer una apertura radical, una verdadera compenetración con la otredad y la alteridad. El neologismo de extimidad es particularmente interesante a este propósito. Para el psiquiatra y psicoanalista J. Tisseron, no es un término 
antagónico de la intimidad sino más bien una forma de la intimidad:

Je propose d'appeler "extimité" le mouvement qui pousse chacun à mettre en avant une partie de sa vie intime, autant physique que psychique. [...] Mais ce mouvement serait incompréhensible s'il ne s'agissait que "d'exprimer". Si les gens veulent extérioriser certains éléments de leur vie, c'est pour mieux se les approprier [...]. L'expression du soi intime - que nous avons désigné sous le nom "d'extimité" - entre ainsi au service de la création d'une intimité plus riche (Tisseron, 2001: 52).

La extimidad es esa parte de la intimidad que exponemos, desplazando hacia afuera las fronteras de lo íntimo: no se trata de un concepto que anula la intimidad sino que la amplía en exacta concordancia con el sujeto que, desde su cuarto propio conectado, dibuja nuevas fronteras, no precisamente para desvelar o exponer su intimidad sino para enriquecerla de la pluralidad constitutiva de la conexión.

Esa ductilidad de las fronteras dentro/fuera acarrea varias consecuencias, siendo la más llamativa la vertiente política de la intimidad: "De todos los cuartos propios de mi vida, es en los cuartos conectados donde lo privado se funde literalmente con lo público, y entonces lo político se incrementa" (Zafra, 2010: 56). Por cierto, la intimidad feminista de la netiana que hemos destacado es una de las manifestaciones más frecuentes de la dimensión política de la intimidad conectada. La conceptualización de una intimidad librada de la alternativa dentro/fuera, interioridad/ exterioridad, privado/público, personal/político es también la perspectiva adoptada, aunque con enfoque distinto, por J. L. Pardo: "Ciudad e intimidad son conceptos mutuamente irreductibles pero radicalmente inseparables; allí donde no hay política, no puede haber en sentido estricto intimidad; y allí donde la intimidad está amenazada, estas amenazas expresan una crisis del espacio civil" (Pardo, 1998: 147). La intimidad no solamente es una experiencia importante en la configuración de la subjetividad: tiene

5Propongo llamar "extimidad" al movimiento que empuja a todos a presentar una parte de su vida, tanto física como psíquica. [...] Pero este movimiento sería incomprensible si solo se tratara de "expresarse". Si la gente desea exteriorizar ciertos elementos de su vida, es para mejor apropiárselos [...]. El deseo de extimidad, en realidad, está al servicio de la creación de una intimidad más rica. (La traducción es nuestra) 
estrecha relación con el modo en que los sujetos experimentan lo público. Es interesante observar que Un cuarto propio conectado se sitúa en la estela de revisión del paradigma interioridad/exterioridad, en la medida en que insiste específicamente en una dinámica de interacción "centrípeta", en la que el cuarto propio es caja de resonancia de la exterioridad al mismo tiempo que espacio de re-configuración de la interioridad:

Es en su conversión en espacio conectado donde esta nueva fuerza centrípeta (hacia los espacios de intimidad) empieza a operar. Pasa entonces que el clásico escenario de seguridad ontológica por excelencia se transforma, y lo hace en un lugar potencialmente rupturista para la constitución y gestión colectiva y personal (Zafra, 2010: 24).

La dimensión conectada del cuarto no solo confirma y consolida la porosidad entre lo íntimo y lo político, lo privado y lo público, el dentro y el fuera, sino que determina una dinámica centrípeta que otorga un nuevo estatuto al yo, un estatuto céntrico. El texto formula y reivindica una verdadera reactivación del yo en su dimensión íntima como fuente potencial de "gestión colectiva y personal". En este dispositivo, el yo aparece como instancia de incontestable valor ontológico y de fuerte poder político; "habita Internet", recuperando el topos del cuarto propio de Woolf, como espacio de una construcción de una intimidad conectada y, por consiguiente política, susceptible, en particular, de actuar sobre las relaciones de género.

\section{CUENTO DEL CUARTO PROPIO CONECTADO Y CARTOGRAFÍA DE LA INTIMIDAD}

R. Zafra propone por consiguiente una actualización - una "resemantización" (Zafra, 2010: 12)— del espacio de intimidad defendido por V. Woolf (y otras). Desarrolla la propuesta de un espacio propio pero conectado, capaz de resignificar la tradicional dicotomía entre esfera pública/privada y de revitalizar la potencialidad emancipadora del yo femenino. Este proceso de "resemantización" se inscribe en un mecanismo discursivo en el que la reflexión toma la forma de un cuento, centrado en "cómo el cuarto propio pasó a ser el cuarto propio conectado", a partir 
de la experiencia claramente autobiográfica: se va hilvanando la historia de un sujeto de cuento autobiográfico, y detrás, la de una sociedad entera que accede a un espacio revolucionado (¿revolucionario?), digitalizado e irremediablemente conectado.

El cuento empieza en el primer capítulo, "capítulo posición Escritura situada: el yo que habla desde un cuarto propio conectado": "Ahora-Aquí. Entre la cocina y la ventana quince pies de cuarto propio y cuatro estanterías. Enfrente mi ordenador. Aquí es relativo. Aquí es donde estoy conectada y donde la escritura emerge" (Zafra, 2010: 19). Esta apertura textual nos proyecta en un cuarto propio efectivo, presentificado (esencialmente por el presente enunciativo y la presencia de los deícticos "aquí", "ahora"), en el cual el yo femenino habla y escribe: en este sentido, este primer capítulo es la feliz continuación del texto de Woolf. La primera persona remite a una narradora-autora auto-representada en un espacio atentamente descrito en sus detalles más "vivenciales", con "silla a ser posible mullida (vale sillón, cojín o sofá), mi portátil, módem y yo”. Sin embargo, este mismo espacio es presentado como "relativo", sometido a la autoridad de un sujeto autobiográfico ("y yo") celebrado como el componente álgido de la retahíla descriptiva: un yo que, literalmente, "construye" su cuarto como si fuera "una madriguera o un nido", un yo arquitecto de un espacio perfectamente materializado y determinado, pero cambiante y mutante:

Les habla un yo conectado que habita cada vez más en su cuarto propio. [...] Así, hablaré desde la legitimidad del yo que, entretejido de identidades de época, se sabe (se quiere) agente de sus palabras, incluso cuando sus palabras son cita, apropiación o parodia de otras. Quisiera con ello llegar al mundo a través del propio, advirtiendo de prejuicios posibles, defendiendo la experiencia, lecturas y motivaciones de uno mismo como un argumento más de autoridad [...]. En consecuencia, debo decirles que existo (Zafra, 2010: 12-14).

En estas aseveraciones programáticas y contundentes, lo que se impone es la reivindicación de un yo conectado, por una parte, y "cada vez más en su cuarto propio", cada vez más en su intimidad, por otra parte. La totalidad de la cita se apoya en un sutil vaivén entre el yo en red y el yo en 
el cuarto, el yo tautológico ("hablaré desde la legitimidad del yo") y el yo plural ("entretejido de identidades de época"), el yo totalmente autónomo en su toma de palabra ("agente de sus palabras") y el yo plenamente ocupado por las palabras ajenas "entretejido de identidades de época"). Por fin, se impone un yo que pretende "llegar al mundo", pero a partir de su "propio mundo", como clara prefiguración de una intimidad que deja de ser un espacio de retraimiento para convertirse en punto de partida de exploración y de renovación del afuera, en centro que permite llegar al mundo. El espacio propio conectado, la intimidad conectada condiciona en cierta medida, la inteligibilidad del mundo "ancho y ajeno", prefigurando la dinámica centrípeta propia de las intimidades conectadas.

Después de esos preliminares, el texto entra en los pormenores de la trama narrativa. El primer episodio, lo constituye el cuarto propio tradicional, primigenio, lo que da pie a la vertiente más auténticamente autobiográfica del texto. El capítulo titulado "capítulo material o la construcción del cuarto propio", y dentro de él, la secuencia titulada "Mi primer cuarto propio", nos ubican en la intimidad de las autobiografías tradicionales:

Mi primer cuarto propio habla de la infancia. Mi primer cuarto propio estaba en una casa antigua y grande que a su vez estaba en un minúsculo pueblo agrario donde casi todo, salvo los dormitorios, eran espacios comunes o de tránsito [...]. Espacios gestionados por mi madre que, como otras muchachas, nunca tuvo cuarto propio (Zafra, 2010: 35).

Esa intimidad autobiográfica va configurándose mediante la elaboración de un mapa doméstico cuyas fronteras evolucionan a medida que pasa el tiempo de la infancia y de la adolescencia. El esbozo de un nuevo cuarto propio con máquina de escribir y unas estanterías de libros marca una segunda pauta en la reconfiguración del mapa (Zafra, 2010: 37). El tema de la biblioteca paterna (en realidad sencillas "estanterías" albergadas en el cuarto de la hija) es particularmente interesante: es una biblioteca sometida a una ordenación aleatoria y fantasiosa totalmente alejada de cualquier requisito de calidad literaria. Los libros no son sino elementos susceptibles de configurar, delimitar el espacio propio, como lo harían tabiques de ladrillos. Sin embargo, ese nuevo espacio que va 
emergiendo en el mapa mental de la autora, al mismo tiempo que en la cartografía del texto, sigue obedeciendo a la lógica de apertura. Estar dentro del cuarto no es excluyente de estar fuera, precisamente porque los libros que hacían de paredes "apuntaban fuera":

En aquella época, los libros fueron tomando posiciones y las funciones de algunos de los muebles de mi cuarto propio cambiaron, mutando hacia un particular microcosmos. Estando dentro era como estar fuera de la casa, porque afuera apuntaban casi todos los libros (Zafra, 2010: 38).

El espacio propio, la intimidad que la autora, con ayuda de su padre, había constituido, casi construido, sigue siendo un espacio dinámico, un microcosmo protector que "apunta hacia afuera", un dentro que conduce al fuera.

El último cambio que marca la evolución de la cartografía del cuarto propio es fundamental y perfectamente ilustrativo de la peculiaridad de las intimidades y de los espacios propios conectados. Proviene de la irrupción del ordenador, verdadero clímax narrativo que invierte claramente la dinámica que anima el cuarto propio y que impone nuevas coordenadas en la concepción y en la lectura de los mapas de la intimidad:

Llegaron otras paredes y otras ciudades, cristales y muebles variados. Logré cambiar la máquina Olivetti por un modesto pero asombroso ordenador. Y ahi fue cuando los ceros y los unos llegaron para quedarse. Con la conexión de la red, vinieron infinitas historias y todos sus protagonistas con sus particulares colecciones de cosas propias. Tuve entonces que deshacer mis viejos cuartos, "construir $y$ destruir máquinas, identidades, categorias, revelaciones, historias del espacio" [Donna Haraway, Manifiesto para Cyborgs: ciencia, tecnología y feminismo a finales del siglo XX, 1991] (Zafra, 2010: $38)$.

Se va esbozando un espacio propio animado de otra dinámica, un espacio que ya no "apunta" hacia afuera, sino hacia dentro, acogiendo otras historias, otros cuentos de nunca acabar, otros huéspedes (las cifras) que "llegaron para quedarse". Las fronteras delineadas implican por 
consiguiente esa dinámica centrípeta que ya comprobamos, convirtiéndose el cuarto propio en centro estratégico que acoge lo que está fuera. La consecuencia de esta revolución, de esa inversión, es la necesidad, apuntada por D. Haraway, de "deshacer los viejos cuartos", de cartografiar los nuevos en un mapa que esté en coherencia con las nuevas "historias del espacio": historias y/o Historia(s) con toda la riqueza de la ambigüedad semántica del término. Con la digitalización, el paradigma espacial cambia tanto a nivel individual como a nivel colectivo: las habitantes de los nuevos cuartos propios tienen que inventar, fabricar otras $\mathrm{H} / \mathrm{historias,} \mathrm{en}$ adecuación con la fuerza centrípeta que rige — para bien o para mal — los espacios conectados. El desenlace del cuento consagra el final de una labor cartográfica que delimita un cuarto propio conectado, centralizado por el yo, reducido pero dotado de un poder unilateral de atracción. El cuarto propio, y dentro de él, el yo, no se proyectan hacia fuera, sino que fagocitan el fuera, para absorberlo en una dinámica de invención, de renovación y de creación. No es solo el espacio de la auto-reflexión. Es también un espacio concebido por y para un yo céntrico que inventa el mundo al mismo tiempo que se configura en su intimidad:

Sigo en mi cuarto propio. Son las 23.30. [...]; en el cuarto propio conectado: privacidad sin recluirnos del todo. Porque la habitación conectada abriga [...], podemos concentrarnos por fin, y descansar de la dispersión del afuera. [...] Si algo puedo aqui donde construyo mi cuarto propio conectado es idear un espacio para el pensamiento, aquel requerido por la autoconciencia y, más allá, para el trabajo creativo. Es el cuarto propio conectado ese lugar para el silencio donde ahora resuenan muchas voces reguladas, voces que no solamente podemos gestionar [...] sino a las que podemos sumarnos produciendo nuestra voz pública en la Red (Zafra, 2010: 32).

La frase lapidaria, "Privacidad sin recluirnos del todo", es un motivo recurrente a lo largo del texto, específicamente en el capítulo titulado "Volver/estar en casa" que elogia la privacidad, la vuelta y la estancia en un espacio propio, un espacio de "auto-conciencia" que asimila e incluye el fuera en su actividad autorreflexiva. El capítulo rehabilita claramente un "yo-cuerpo-centro de operación", muy alejado de ese yo improbable, fragmentado y disperso que, desde décadas, se impone en 
tantos pensamientos y creaciones posmodernos y contemporáneos. Destaca la posibilidad (y la necesidad) de una "privacidad" centralizadora que no sea exclusiva de una implicación en la esfera pública. El yo "existe" y se construye a través de la edificación de un espacio íntimo conectado, dotado de una fuerza inclusiva (centrípeta), acogiendo las voces para unirse con ellas: habla desde la privacidad de la intimidad, pero su voz es de tesitura colectiva. Su intimidad implica lo colectivo, feliz desenlace del cuento que reúne dimensiones incompatibles, puesto que el cuarto propio conectado ofrece la "posibilidad de ser en el mundo, estando en casa", posibilidad que la narradora considera como "absolutamente transgresora": "ser en el mundo" o sea, asegurar una presencia en el mundo, una huella de su intimidad esencial, propia, inconfundible, pero implicada en la colectividad y, por eso, creadora; "estar en casa", o sea, habitar un espacio que permita, que implique la actualización y fabricación de la intimidad del yo, una intimidad existencial y dinámica, en constante mutación, como condición de una implicación en el mundo controlada, reflexiva y creadora.

En la perspectiva de R. Zafra, si este espacio, en su nueva configuración, se presenta como espacio de autorreflexión, de construcción de sí en tanto que crisol de voces e identidades ajenas, es porque, mediante la digitalización, las funciones de las fronteras dentro/fuera evolucionan: más que de disyunción, son sinónimas de interacción. Tal interacción, tal porosidad no desembocan en la sobreexposición incómoda, cuando no "pornográfica", que podemos observar en las redes sociales y que la propia R. Zafra identifica (y crítica) claramente. La intimidad conectada es una intimidad compartida y, en cierta medida, colectiva, no precisamente porque "se pasea online, deriva, deja rastros" (Zafra, 2010: 44), sino porque esos "rastros" virtuales que ha dejado, que ya no son exclusivamente suyos, son los componentes que le permiten configurar un espacio propio y singular por la red. Esta fábrica de la intimidad no obedece a un movimiento de perdición, dispersión, sino de concentración, de recuperación de elementos heterogéneos, exógenos para reforzar al yo en una intimidad dinámica, plural, abierta y, sin embargo, singular. El yo conectado, y particularmente el yo conectado de la netiana, constituyen, delimitan, en el mapa virtual que va elaborándose, un auténtico lugar, constitutivo de esa intimidad online: "La primera cuestión a tener en cuenta en un ejercicio de actualización del cuarto propio es que éste se concibe como un lugar inscrito, pero diferenciado, del lugar-hogar en que se inserta" (Zafra, 2010: 52). 
El remate final de la trasmutación del cuarto propio es, efectivamente, su consagración como lugar, en el sentido antropológico que le da Marc Augé, explícitamente citado por R. Zafra en el capítulo "La habitación propia como lugar (no) Doméstico". En Non-lieux. Introduction à une anthropologie de la surmodernité, M. Augé establece una distinción fundamental entre la noción de espacio y la de lugar ${ }^{6}$. Un espacio puede ser considerado como lugar a partir del momento en que cumple con tres requisitos: "es identificatorio", proporciona una serie de datos y fenómenos que son referencias identificatorias para el sujeto, permitiéndole reconocerse y ser reconocido en virtud de ese lugar; "es relacional", en el sentido de que su configuración se apoya necesariamente en un sistema de relaciones compartidas por las/los que transitan por él; "es histórico", y se inscribe en una línea temporal, en una filiación y un proceso de transmisión de orden privado o colectivo. Según R. Zafra, el cuarto propio conectado cumple - o tendría que cumplir para realizarse como tal — con estos tres requisitos. Es primero un espacio referencial identificatorio, cuya cartografía traduce la singularidad del sujeto conectado: "Si el itinerario es anónimo, casual y fruto de la deriva, estaremos en un espacio, si nos identificamos y subjetivamos, convirtiéndonos en referencia, transitaremos por un lugar" (Zafra, 2010: 42). Esta construcción, este mapa, nos dice R. Zafra, se inscribe en una historia, nutriéndose del big data personal del sujeto, de su historia propia: "De muchas maneras este sentimiento de posesión y cercanía también existe online, en los lugares emocionalmente cargados con fragmentos de nuestro yo como escaparates de subjetividad" (Zafra, 2010: 42). Por fin, este espacio singular es, sin embargo, intrínsecamente relacional, conectado: "En esta línea, la singularidad de este cuarto propio conectado viene significada por la intersección permanente con las visiones y los mapas simbólicos de los otros" (Zafra, 2010: 46). En definitiva, la trayectoria virtual del sujeto delinea los contornos de un mapa cognitivo individual, que linda con otros mapas con los que interactúa. El sujeto que construye su intimidad a partir del cuarto propio conectado convierte este espacio en auténtico lugar, habitado por un yo céntrico, catalizador de todo lo que está fuera, dedicado a una itinerancia aleatoria e imprevisible, interactuando con trayectorias de otros sujetos: un lugar "versátil y

${ }^{6 " C e s ~ l i e u x ~ o n t ~ a u ~ m o i n s ~ t r o i s ~ c a r a c t e ̀ r e s ~ c o m m u n s . ~ I l s ~ s e ~ v e u l e n t ~(o n ~ l e s ~ v e u t) ~ i d e n t i t a i r e s, ~}$ relationnels et historiques" (Augé, 1992: 64). 
movedizo, en territorio adisciplinar" (Zafra, 2010: 56) que permite abordar la cuestión de la intimidad desde un punto de vista dinámico, y no solo tópico.

\section{CONCLUSIÓN: UNAS INTIMIDADES IKEA}

En el cuarto propio conectado, el sujeto en su dimensión íntima es el fruto de una verdadera fabricación que R. Zafra asimila, con humor, al paradigma IKEA. En este dispositivo, el sujeto no descubre una intimidad ya configurada, latente, sino que la fabrica como si tuviera, como punto de referencia, una imagen preconcebida de cuarto propio amueblado (la de los catálogos o páginas web de IKEA) de la que se deriva la compra de los fragmentos para construirlo: compra unos elementos ya hechos, pero los combina para componer un espacio propio más o menos singular, más o menos propio. En otros términos, consume productos que forman parte de un conjunto normativo y canónico (normas, ideales de moda, calidad etc.), integrando el nosotros, pero también genera los contenidos que los hacen singulares, fabricando lo que le singulariza en su dimensión íntima.

La intimidad conectada consiste en ese sutil equilibrio entre un yo que tiene que ser preponderante (pero no omnipresente), un "yo real", y un nosotros en segundo plano (pero no ausente), un "nosotros ligero" (Zafra, 2010: 73). Más allá del humor de la comparación y de una bienvenida desmitificación del concepto de intimidad, esa parábola nos da a entender que la presencia del nosotros es esencial. En este caso, no significa solo la alteridad, sino más bien la pluralidad: la intimidad no puede concebirse sin la pluralidad de la que formamos parte. Sin embargo, para que lo íntimo pueda surgir, para que tenga alguna realidad, para que habite el espacio y lo configure, el nosotros tiene una presencia ligera. Ese "yo real", ese yo que "existe", se realiza aligerándose del nosotros, realizando, fabricando una intimidad mestizada en y por el cuarto propio conectado y a través de sus peregrinaciones virtuales: la intimidad conectada resulta así de una verdadera manipulación, sin ser forzosamente por eso inauténtica.

En esta perspectiva, es interesante hacer referencia al subtítulo de la obra: Un cuarto propio conectado. (Ciber)espacio y (auto)gestión del yo. Podemos entender el término gestión como diligencia, trámite necesario para conseguir algo o resolver un asunto, habitualmente de carácter administrativo: es un término que designa un conjunto de operaciones que 
requieren procesos de planificación, desarrollo, implementación y control. Gestionar el yo, tramitarlo, nos indica que la intimidad solo puede emerger a raíz de una serie de operaciones reflexivas, inventivas y creativas, a partir de la constitución paciente de un territorio virtual familiar e identificatorio, dinámico y compartido. Ese cuarto propio conectado se asimila, en este sentido, con lo que sería una utopía contemporánea de una intimidad concebida como núcleo más profundo de nuestra subjetividad y, a la vez, como dimensión plenamente insertada en el espacio público. El texto de R. Zafra privilegia la visión del cuarto propio conectado como laboratorio, como fábrica de una intimidad virtual auténtica y transgresora, fundadora, sin embargo, de un yo real y situado: la propuesta es ambiciosa, pero necesaria en un contexto de digitalización creciente, irreversible en el que el sujeto, y más particularmente el sujeto femenino, tiene que encontrar su lugar.

\section{REFERENCIAS BIBLIOGRÁFICAS}

AUGE, M. (1992). Non-lieux. Introduction à une anthropologie de la surmodernité. Paris: Seuil.

JANKELEVITCH, J. (1979). L'ironie. Paris: Flammarion.

PARDO, J. L. (1998). "Política de la intimidad: Ensayo sobre la falta de excepciones”. Logos. Anales del Seminario de Metafísica 32, 145 196.

TISSERON, S. (2001). L'intimité surexposée. Paris: Éditions Ramsay.

ZAFRA, R. (2005a). Netianas. N(h)acer mujer en Internet. Madrid: Lengua de Trapo.

(2005b). "N(h)acer mujer en Internet: Entrevista a Remedios Zafra". Mujeres en red. El Periódico Feminista, http://www.mujeresenred. net/spip.php?article154 [07/02/2019]. (2010). Un cuarto propio conectado. Madrid: Ediciones Fórcola.

Recibido el 5 de abril de 2019.

Aceptado el 3 de junio de 2019. 